\title{
La Construction de L’Opinion Publique Turque sur Hamas: Un Traite Par le Contenu Médiatique
}

\author{
Hamas Hakkında Türk Kamuoyunun İnşası: \\ Medyatik İçerik Üzerinden Bir Değerlendirme
}

\section{Tuğçe ERSOY CEYLAN *}

Résumé: Les conflits internationaux se sont transformés aujourd'hui non seulement en des batailles qui sont faits avec des armes mais aussi des batailles des opinions. La propagande joue un rôle important en engendrant les camps du conflit. L'application des stratégies de propagande par l'entremise de media démontre que c'est le media qui est la première source pour accéder à l'information. C'est la raison pour laquelle les recherches qui sont faits sur la représentation des événements, des crises ou des guerres sont importantes afin de comprendre le processus de formation des opinions et de l'opinion publique. Pour mieux comprendre le rôle du media, il faut examiner les messages donnés, la structure du contenu de l'information et le contexte de l'information. Ainsi, le contenu du texte de l'information, sa manchette et sa structure influencent et orientent le commentaire de l'audience. Dans cette étude, on essayera montrer qu'il existe un lien entre les cadres contournés par le media et l'opinion du public sur ces sujets parce que le media n'a pas seulement la fonction d'informer mais aussi d'influencer. Dans ce contexte, le sujet du traitement dans cette étude sera le Hamas, le vainqueur des élections Palestiniens en 2006. Le but de cette étude est de trouver un lien théorique entre le media et ses effets sur la formation de l'opinion publique et après, de révéler quelle sorte de l'opinion public se forme à propos de Hamas en examinant la façon de représentation de Hamas dans trois journaux turcs, Hürriyet, Birgün et Radikal en appliquant l'analyse critique du discours de Teun Van Dijk comme le cadre d’analyse.

Mots-clés: Hamas, Opinion Publique, Analyse Critique du Discours, Les Élections Palestiniennes de 2006

Öz: Uluslararası uyuşmazlıklar günümüzde silahlarla yapılan savaşlar ile birlikte, düşüncelerin de kullanıldığı savaşlar haline gelmiştir. Uyuşmazlığın taraflarını en iyi şekilde çerçevelemede propaganda önemli bir role sahiptir. Günümüzün dünyasında kişilerin bilgiye ulaşmasında medyanın birincil kaynak olması, propaganda stratejilerinin medya aracılı̆̆ı ile uygulanma nedenini açıklamaktadır. Bu yüzden, olayların, krizlerin ya da savaşların medyada temsili üzerinde yapılan çalışmalar düşüncelerin, daha genel anlamda kamuoyunun oluşma sürecini anlamayı sağlamaları açısından önemlidir. Medyanın rolünü daha iyi anlamak için, medyada konu ile ilgili verilen mesajlara, haberin içerik yapısına, bağlamına bakmak gereklidir. Nitekim haberin içeriği, manşeti ve yapısı izleyicinin olayı yorumlamasını etkilemektedir. Bu çalışmada medyanın gündeme taşıdığı sorunlar için çizdiği çerçeveler ile halkın bu sorunlar hakkındaki görüşleri arasında bir bağ olduğu gösterilmeye çalışılacaktır. Zira medyanın sadece bilgilendirme değil, etkileme işlevi de vardır. Bu çerçevede bu çalışmada ele alınan aktör 2006 Filistin seçimlerinden galip çıkan Hamas'tır. Çalışmanın amacı medya ve kamuoyu oluşumu arasındaki teorik bağı bulmak ve sonra Teun Van Dijk'in eleştirel söylem analizi yaklaşımını kullanarak üç Türk gazetesinde, Hürriyet, Birgün ve Radikal, seçimler hakkında çıkan haberleri analiz ederek Hamas hakkında nasıl bir kamuoyu oluştuğunu ortaya çıkarmaktır.

\section{Anahtar sözcükler: Hamas, Kamuoyu, Eleştirel Söylem Analizi, 2006 Filistin Seçimleri}

\footnotetext{
* Maître de conférences adjoint, Université İzmir Demokrasi, Département des Relations Internationales, İzmir. tugce.ersoy@idu.edu.tr, https://orcid.org/0000-0001-5478-3539
} 


\section{Introduction}

Les conflits internationaux se sont transformés aujourd'hui non seulement en des batailles qui sont faits avec des armes mais aussi des batailles des opinions. Dans ce contexte, c'est la propagande qui joue un rôle important en engendrant les camps du conflit. L'application des stratégies de propagande par l'entremise de media démontre que c'est le media qui est la première source pour accéder à l'information. C'est la raison pour laquelle les recherches qui sont faits sur la représentation des événements, des crises ou des guerres sont importantes afin de comprendre le processus de formation des opinions et de l'opinion publique.

La façon dont le média couvre les nouvelles influence la formation de nos perspectives, nos perceptions, nos opinions à la manière dont ils sont reflétés par le media. Les recherches de représentation des informations au media s'appuie sur l'acceptation par la plupart des chercheurs de communication que le media a des effets sur l'audience, sur la société dans laquelle on vit. Diverses théories sont proposées pour expliquer les effets de media. Les effets de media qui sont relevés par ces théories ont une place importante dans le contexte de représentation des événements et des effets sur l'audience, particulièrement en reflétant les groups qui sont en conflit.

Le conflit qui mène depuis plus de cent ans et qui est connu comme «Le Problème de Palestine » ou « Le Conflit Israélo-palestinien » est l'un des issues dont le media couvre le plus. Le media est devenu la plus importante arme des deux camps; chaque camp a essayé de mettre l'autre à une situation désavantageuse dans les yeux du public par l'intermédiaire des organes du media. La même situation prévalait dans le media international ce qui couvrit des nouvelles à propos du Problème de Palestine.

Dans cette étude, on essayera de montrer qu'il existe un lien entre les informations choisi par le media pour couverture et la création de l'opinion du public sur ces issues concernées. Dans ce contexte, on essayera de trouver quel sort de l'opinion publique a été formée à propos du Hamas, le vainqueur des élections Palestiniens en 2006 en Turquie en examinant trois journaux turcs, Hürriyet, Birgün et Radikal. Le but de cette étude est de démontrer que le media a des effets sur la formation de l'opinion publique Turque et que la façon de représentation du Hamas dans certaines parties de la presse Turque forme une opinion publique négative sur le Hamas. Pour parvenir à ce but, on examinera les informations médiatiques sur les élections parus durant une semaine dans ces trois journaux turcs.

C'est la contention de cette étude que le media n'ont pas seulement la fonction d'informer mais aussi d'influencer. Donc, il a un effet fort en formation de l'opinion publique. Dans ce cadre il est possible d'exciper que les attitudes vis-à-vis le Hamas sont construits par rapport à la façon de couvertures des journaux sur le Hamas. Le plus important déterminant de cette approche est les tendances politiques des journaux. Suivant ces tendances, une opinion publique à propos du Hamas, soi négative soi positive est formée. Donc, on peut proposer que la façon de présentation du sujet ait une influence forte sur la formation de l'opinion publique.

Sur le fond de cette argumentation, on analysera la couverture des nouvelles sur le Hamas comme le vainqueur des élections de 2006 en Palestine. La semaine des élections, y compris le 23 Janvier 2006 et le 30 Janvier 2006 est choisi pour l'analyse des données. Les journaux dont on examinera sont déterminés selon leurs positions politique. À ce compte, on a examiné Birgün comme le journal du gauche, Radikal libéral, Hürriyet au centre droit. On va essayer de montrer comment ces trois journaux ont couvert les nouvelles sur le Hamas en appliquant l'analyse critique du discours qui a été évaluée par Teun A. Van Dijk.

L'analyse se compose de trois parties. La première partie se compose de la définition théorique; dans la deuxième partie le sujet de l'étude, le Hamas sera examiné par son arrièreplan historique et politique. Finalement, on analysera les données dont on a acquis des journaux 
qui sont en traitement selon le modèle de Van Dijk.

\section{Le Cadre Analytique: L’Analyse du Discours}

L'analyse du discours a acquiert une croissant proéminence dans les recherches qualitatives dans les dernières années. Elle est un nouveau champ d'étude interdisciplinaire qui se dégage de plusieurs autres disciplines des sciences humaines et sociales telles que la linguistique, études littéraires, anthropologie, sémiotiques, sociologies, psychologie et la communication de parole (Van Dijk 1988a, 17).

Le terme discours renvoi à manifestations concrètes du langage et implique donc une prise en considération du locuteur, du référent et de la situation de communication. Cependant, chaque école linguistique lui donne un sens légèrement différent (Detrie et al. 2001, 168). Stuart Hall suggère la définition suivant pour le discours: "ensembles des expériences réalisés et préconstitués qui sont affichées et arrangées par le langage » (Purvis \& Hunt 1993, 485). Le concept tente de capturer que les gens vivent et font l'expérience au sein du discours. Les discours, de cette manière en imposant des cadres qui limitent les sens de l'expérience, influence ce qui est dit ou fait. Par les discours certaines choses peuvent être dites et certaines choses sont empêchées d'être dites. Par conséquent les discours deviennent des moyens qui ont des influences spécifiques dans l'acte de communication.

Dans les théories critiques de media, l'information n'est pas un instrument qui reflète la réalité. Elle est considérée comme le discours qui reconstitue la réalité. Les textes représentent et reflètent non seulement une certaine version de réalité, mais aussi ils prennent le rôle dans la construction et la maintenance de réalité, elle-même. Il existe une relation dynamique entre le texte et contexte dans lequel le texte est produit. Les textes sont constitutifs et aussi sont construit par leurs contextes (Cheek 2004, 1144). L'œuvre du Gaye Tuchman, Making News: A Study in the Construction of Reality (1978) pourrait être compté comme le premier exemple important pour l'hypothèse que l'information construit la réalité.

Tuchman (1978) voit l'information comme un cadre et il cherche à répondre comment cet encadrement est construit par les agences d'information et les journalistes. Cette approche accepte l'information comme une institution qui est située à la vie quotidienne, y compris à la réalité qu'elle construit. Par conséquent, pour Tuchman; avec la logique de fonctionnement et sa structure, ces institutions de l'information déterminent l'histoire et la narration de l'information. Donc, l'information est présentée comme les représentations de réalité qui a l'occasion de manipuler la réalité. Tuchman définit l'information comme un encadrement en partant de théorie de Goffman. Les particularités des agences d'information déterminent la formation d'encadrement des informations. Ces encadrements limitent la circulation des sens sociaux et ces sens sociaux limités construire la réalité sociale (Dursun 2004, 3-43). Cette construction est réalisée par les intermédiaires que désigne Van Dijk «le pouvoir social ». Le pouvoir social est une relation entre les groupes, les classes ou bien les autres formations sociales. En outre, il signe aussi les relations interpersonnelles comme les membres d'une société. Van Dijk (1988) souligne que le pouvoir social trouve sa force en dirigeant/contrôlant les informations et les opinions à l'occasion de son intérêt.

Pour Van Dijk, afin de contrôler les opinions; le pouvoir social s'adresse au discours: il reproduit le discours et par là, il le contrôle. Ce contrôle put se réaliser par plusieurs façons: par les formes variables de reproduction du discours, par l'articulation, par la distribution et par la base de l'impact. Dans cet article, Van Dijk, en partant par Bourdieu, suggère que la façon de production est contrôlée par "les élites symboliques » telles que les journalistes, les écrivains, les directeurs et les académiciens. Ce pouvoir symbolique formé par les élites symboliques n'est pas non seulement limité par l'articulation mais aussi il se prolonge jusqu'aux sortes de l'impact sur les individus. Ces élites peuvent former l'agenda du public, ils décident lequel titre sera 
utilisé dans les journaux, ils décident aussi laquelle information sera étendue publiquement avec quel style. Par conséquent, Van Dijk parvient que ces groupes sont les producteurs des croyances, des attitudes, des normes sociaux, des informations publiques et en fin des idéologies. Donc, ce pouvoir symbolique est en même temps une sorte du pouvoir idéologique (Van Dijk 2005, 315-22).

Il est possible de dire que le media de masse joue un rôle important dans la production des opinions. Les groupes ou les institutions puissantes pour ses propres intérêts manipulent l'information en excipant des raisons économiques ou politiques. L'une des formes de manipulation se compose de contrôler la compréhension: en imprimant une partie du texte, en utilisant les sources éclectiques, en transmettant les informations dans un tempo monotone et en choisissant le titre de la nouvelle; le media décide quels acteurs seront représentés au public, qu'est-ce que seront dits à propos d'eux et le plus important comment ces choses seront dites (Van Dijk 2005, 350). Cette stratégie a été témoignée dans le processus de légitimation de l'attaque en Iraq en 2003: «La connaissance » qu'il existe les armes de destruction massive en Iraq s'est révélée fausse. Plus on a beaucoup d'information à compter, plus on subit à manipulation de media.

Une fois que les attitudes de la population sont influencées, par exemple sur le terrorisme, peu ou pas des tentatives manipulatoires peuvent être nécessaire pour que les gens agissent en fonction de ces attitudes, par exemple, de voter en faveur de politiques anti-terroristes (Van Dijk 2006, 369). Il est maintenant possible de dire que l'information est formée par la structuration des médias, elle est produite par les classes dominants dans la société à travers leurs propres intérêts; c'est la raison pour laquelle, elle a une dimension manipulatoire. Cette structure peut être expliquée plutôt par les analyses qualitatives. La méthode d'analyse du discours de Van Dijk est l'une des méthodes le plus appliquée comme une analyse qualitative.

L'analyse du discours de Van Dijk replace les textes dans leur contexte social, culturel, politique et historique et fait poser les questions suivantes: «Pourquoi ceci a été dit, et pas cela ? « Pourquoi avec ces mots? » (Cheek 2004, 1145).

\section{L’Analyse Critique du Discours et Le Modèle de Van Dijk}

Si l'on prend compte des rôles des médias en formation de l'opinion et l'agenda du public, il est possible d'exciper que les textes informatifs sont différents des autres. L'analyse du discours révèle que le terme a de nombreuses définitions et des approches. Il est cependant tout à fait possible pour un lecteur de discerner que la plupart de ces approches manquent en autonomie et qu'ils peuvent être possiblement replacés (Bekalu 2006, 148).

Dans cette étude, toutes les approches pour l'analyse du discours ne seront pas traitées. Cette étude va concentrer sur les approches du discours médiatique, développé par linguiste Hollandais Teun Van Dijk. L'approche de Van Dijk est importante parce qu'il tente de former une connexion entre l'utilisation de langage et le pratique du pouvoir social. Pour cette raison son travail est marqué comme «l'analyse critique du discours ».

Le discours est compris comme un moyen à travers lequel - et dans lequel - les idéologies sont reproduites. C'est Teun Van Dijk qui a produit une théorie socio-cognitive de l'idéologie en partant du discours. Il applique sa méthode aux textes de media depuis 1980. Il est distingué des autres chercheurs par ses analyses qui examinent les textes de media non seulement sous l'angle textuel et structurels mais aussi sous le niveau de production et compréhension (Ülkü 2004, 374). Selon lui, le discours n'est pas indépendant de production et de l'interprétation; c'est pourquoi il doit être examiné dans le contexte social.

News as Discourse est le premier livre de Van Dijk dans lequel il applique la théorie du discours aux textes de media. En examinant le discours idéologiquement, Van Dijk insiste 
particulièrement sur les cognitions sociales qui sont la conscience, la production de sens et les termes d'intuition. Selon Van Dijk, la plupart des connaissances politiques et idéologiques viennent par le media. Ces textes de media reproduisent le sens qui a des effets idéologiques. Donc, l'opinion des individus est formée par ces textes idéologiques.

Van Dijk est intéressé tout d'abord avec le sens de l'information. Il fait un travail holistique, autrement dit il intègre les analyses qualitatives et quantitatives. Il met en lumière les effets idéologiques et sociaux de l'information en la transformant à un texte. L'information est évidemment une sorte très particulière du texte. Les articles sont généralement petits, isolés et autonomes. C’est la raison pour laquelle les articles sont sélectivement décontextualisés. Ils peuvent être recontextualisés par le lecteur mais seulement avec l'aide des idées reçus (Ginneken 1998, 155). Dans son œuvre, Van Dijk prend les informations comme une forme du discours. Selon lui, les informations sont les textes qui sont reproduits et construits dans le pouvoir social. Dans son modèle, il distingue deux structures de l'information comme le niveau d'analyse: les micro structures et les macro structures.

L'analyse au niveau de la micro structure se concentre sur l'analyse de syntaxe, de lexique, des rapports significatifs entre les phrases (la cohérence locale) et enfin la rhétorique de l'information. Le but de cette analyse est d'arranger les unités qui assurent la rhétorique comme la présentation de connaissance. Dans l'analyse de micro structures, en faisant l'analyse syntactique Van Dijk commence à mettre en évidence la nature idéologique des informations. La cohérence locale observe les liens entre les parties des phrases qui articulent. Il est possible que les rapports entre les phrases amènent l'analyse aux aperçues idéologique. La lexicalisation est le niveau de la structuration idéologique. Le choix des mots reflète l'idéologie de correspondant en particulier et l'idéologie du journal en général (Van Dijk 1988a, 77-81). L'analyse grammaticale de l'usage de langue dans la presse peut aussi révéler la perspective du journaliste ou le journal. La syntaxe exprime les rôles sémantiques des participants dans un évènement par l'ordre des mots, par les fonctions relationnelles ou par l'usage des formes actives ou passives. Cette situation est évidente particulièrement dans les manchettes:

Une manchette comme "La police a tué le démonstrateur » met la police au premier, à position du sujet et exprime que la police a le rôle de représentant. Pour la forme passive de cette phrase, la police est aussi représentant, mais dans ce cas, le phrase renvoi au démonstrateur au premier, à position du sujet; c'est-à-dire, la police est assignée un rôle moins important. Finalement, la manchette "Démonstrateur est tué» peut faire le rôle de la police implicite. (.....) La recherche grammaticale sur la syntaxe de journal a montré que ceci est le cas: Les rôles négatifs des élites ont tendance à être dissimulé par ce sort des mises dans une catégorie inférieure (Van Dijk 1988b, 11).

Quant à la rhétorique de l'information, il s'agit des citations, des commentaires et des réactions des témoins afin d'augmenter la crédibilité de l'information et de faire convaincre les locuteurs que l'information reflète la réalité. Il est utilisé aussi les photos, les images pour montrer que l'événement a vraiment eu lieu. En bref, les éléments de la rhétorique de l'information peuvent être juxtaposés comme: les graphiques, les nombres, les données numériques, les photos, les images, les citations par témoins (Van Dijk 1988a, 82). Van Dijk distingue la macro structure en deux: l'analyse schématique et l'analyse thématique. Il observe dans la structure thématique le surtitre, le titre, sous-titre, le spot et les paragraphes d'introduction (lead). La structure schématique reflète les catégories du texte de l'information. C'est une superstructure qui construit le squelette du texte de l'information. L'ordre des catégories est assuré par les corrélations entre les catégories. Les informations le plus générales se trouvent dans résumé, la 
manchette et dans le paragraphe de l'introduction.

L'analyse du discours serve de susciter les sens cachés derrière le texte. L'analyse critique du discours, quoique, vise d'évaluer les discours qui déterminent nos croyances, nos attitudes et nos actions dans un contexte social. Les textes de media n’ont aucune différence des autres produits. Ils sont les produits de media qui vise augmenter son profit. Donc, le processus de production des informations est un processus dans lequel le monde est reproduit d'après les intérêts de ceux qui ont le pouvoir. Donc les travaux sur les textes d'information essayent de reflète l'effet de cette production sur les masses.

Le but fondamental de l'analyse du discours est de pouvoir commenter et pouvoir donner le sens aux textes de l'information. Quant à l'analyse critique du discours, le discours est une pratique sociale qui représente le monde; il devient significatif dans le contexte social et idéologique. Cette analyse intéresse plutôt à production du pouvoir, y compris les idéologies et sa reproduction par le discours.

\section{Le Contexte Historique Dans Lequel Le Hamas S'est Elevé}

Tout au long d'histoire, aucune formation ne s'est révélée inopinément. Une telle formation est préparée par certaines dynamiques qui entrent interaction dans le processus historique. Les accumulations qui se sont produites pendant ce processus forment en fin une formation sociale. Chaque formation, mouvement, organisation sont influencés par leurs endroit qui les entoure et ils ne peuvent pas être compris sans tenir compte les conditions sociales, économiques et culturelles dans lesquelles ils se trouvent.

Depuis la fin des années 1970 l'Islamisme est devenu un composant majeur dans la vie sociale et politique de la plupart des états Arabes (Legrain 1997, 159). Aujourd'hui, elle est devenue une force politique dans la Bande de Gaza et dans la Cisjordanie. Si l'on jette un coup de l'œil au mouvement Palestinien de $20^{\text {ème }}$ siècle, on témoigne que les premiers 87 années de $20^{\text {ème }}$ siècle l'histoire est racontée avec sans référant aux mouvements islamiques. Quant aux derniers 20 années, il est possible de dire que le mouvement Palestinien s'est mis à se définir avec la religion, particulièrement, l'Islamisme.

D’après John Voll (2006) si l'on jette encore un regard aux années 1960 et 1970 du mouvement Palestinien, on verra que la dimension religieuse existait mais qu'elle a été ignorée parce qu'il a été considéré que la religion était en train de disparaître en raison du processus de modernisation. Mais, ce n'était pas le cas.

Depuis le début des années 1970, le Moyen Orient a été confronté avec des crises politiques, économiques et sociales. La croissance rapide de la population, les politiques économiques infructueux, le caractère autoritaire des régimes etc. peuvent être comptés comme les causes probables de cette crise. La croissance rapide de la population a culminé avec le chômage car les gouvernements étaient incapables de fournir des métiers. D’autre part la pauvreté a influencé une grande partie des jeunes. Ces jeunes ont commencé à questionner la légitimité des régimes et conséquemment cette situation les a conduits à joindre aux rangs des mouvements islamiques. Cependant il serait convenable de suggérer que les mouvements islamistes sont nés en raison de la déception des personnes avec l'ordre existant. Les régimes régnants ont généralement été insensibles aux revendications populaires et en conséquence ils n’ont pas réussi à fournir des services sociaux pour la population qui augmente rapidement (Karakır 2006, 86).

Comme Voll signale et comme les raisons mentionnées ci-dessus montrent qu'en réalité Hamas ne s'est révélé pas spontanément dans les territoires Palestiniens occupés. Le mouvement définit une étape de résistance contre l'occupation et une réaction à l'Autorité Palestinienne qui est dégénérée. Le Hamas porte d'une coté les traces du mouvement islamique, Les Frères Musulmans (al-Ikhwan) et l'autre côté les particularités de la question de Palestine. 


\section{La montée du mouvement islamique dans les territoires occupés}

Hamas est une création de Frères Musulmans en Palestine. Les Frères Musulmans est une organisation politique et religieuse fondée en 1928 en l'Egypte par Hasan al Banna ${ }^{\mathrm{i}}$ et bientôt elle a été répandue aux autres parties du monde Arabe. Dans sa tentative de revitaliser l'appel islamique, al Banna a insisté sur trois éléments: la renaissance, l'organisation et l'éducation. La première chose était de fonder l'Etat Islamique avec pas de distinction entre la religion et le gouvernement; la priorité de l'organisation était d'assurer la transformation sociale par l'éducation et la propagande. Djihad serait l'étape postérieure.

La connexion des Frères Musulmans avec Palestine date l'année 1935. Suivant les accroissements en Palestine, al-Banna a envoyé son frère Abd al Rahman al Banna pour qu'il fonde les contacts là-bas. En 1945, la première branche a été ouverte à Jérusalem par Mufti de Jérusalem, Hadjj Amin al Husseini. En 1947, le nombre des branches a augmenté à 25 et les nombres d'adhésion de 12.000 à 20.000 (Abu-Amr 1993, 6). Le but était de protester la présence de Bretagne et de promouvoir la cause Palestinienne.

En 1936, Les Frères Musulmans a participé aux attaques contre les Anglais et les Juifs. Cela est connu comme «La révolte de 1936 ». Après qu’ Israël est fondé en 1948, les volontiers des Frères Musulmans ont combattu contre le nouveau Etat.

La guerre de 1967 (La Guerre de Six Jours) dont les résultats forment l'une des bases du conflit Israélo-palestinien, est inoubliable pour les Palestiniens. La guerre qui a commencé le 5 Juin a mené juste six jours et tous les territoires Palestiniens sont envahis par Israël; Jérusalem, Le Plateau du Golan et Le Sinaï inclus. La Guerre de Six Jours a causé des dommages soi substantiel soi moral; en même temps, elle a fait remonter la conscience nationale Palestinienne et la conscience islamique (Lewitt 2006, 21). Dans cet état d'âme social, la religion est devenue encore une libératrice. Dans les années suivantes de l'occupation, Les Frères Musulmans a emprunté la méthode de résistance désarmée. L’organisation a reconnu dans les activités plutôt morales. Ce ne signifie pas que les membres des Frères Musulmans ne sont pas entrés en affrontement armé; mais les leaders de l'Ikhwan ont donné l'importance, tout d'abord, à transformation sociale et à l'augmentation de la conscience islamique dans la société. Cette attitude a pour l'hypothèse que l'Ikhwan s'est intéressé sur les générations qui combattront contre l'occupation.

Durant les années 1967-1975, le rôle du mouvement islamique a été caractérisé par construction des mosquées, provider aux jeunes des guidances et renforcer leurs idéologies. Pour ces buts, le mouvement a utilisé tous ses moyens et ses dispositions. Le mouvement a connu la force des organisations de charité pour répandre leur message et pour gagner les cœurs et les esprits. Il a fondé les associations de charité islamique qui dirigeait les écoles locales. Il gérait aussi les jardins d'enfants qui étaient attachés aux mosquées. Dans les années suivantes, Les Frères Musulmans et les autres éléments islamiques ont fondé des plusieurs associations islamiques dans Hébron, Jérusalem, Gaza et dans les autres villes Palestiniennes (Abu-Amr 1993, 7-8).

Les centres d'éducation du mouvement étaient généralement les mosquées et les masdjid (petite mosquée). Le futur fondateur et chef spirituel du Hamas, Shaykh Ahmad Yassin a été profondément impliqué dans cette période de floraison de 'dawa'. En 1973, al Mujamma al Islami (Le Centre Islamique) a été fondé par Shaykh Ahmad Yassin qui est devenu après l'institution le plus importante. C'était aussi le premier pas qui a été fait pour la fondation de Hamas. Sauf Shaykh Ahmad Yassin, les noms qui formeraient le cadre des leaders du Hamas, comme Abd al Aziz Rantisi, Mahmoud Zahar, se trouvaient ils aussi dans cette association. En 1981, le Mujamma a créé Jamiyyat al Shabbat al Muslimat (Le Centre Islamiques des Jeunes Femmes) comme l'autre organisation pour l'action sociale et la mobilisation. Dans les années 
1980, le mouvement islamique a gagné beaucoup des partisans dans les universités. Spécifiquement, L’Université Islamique de Gaza est devenue la forteresse du mouvement.

La langueur des Frères Musulmans à propos de résistance organisée provoquait de temps en temps la confrontation entre al Fatah et les Frères Musulmans. Israël a su bien profiter de cette concurrence. Israël a cru que la montée des groupes islamiques serait affaiblie Organisation de Libération de Palestine (OLP). Donc, Israël a commencé à appuyer les activités du mouvement islamique. Ainsi, il a contribué la montée du mouvement islamique en Palestine. Bien plus, le préfet de Gaza, Yitzhak Segev l'a vérifié dans un reportage avec les correspondantes en disant que le gouvernement d'Israël a assuré de l'argent au mouvement islamique contre les communistes et OLP (Bayraktar 2007, 52). C’est pourquoi, le mouvement a été accusé long temps de collaborer avec Israël.

Dès les années 1970, le durcissement des politiques de l'occupation d'Israël et la montée, en Israël des parties politiques d'extrême droit comme Likoud et des parties religieuses comme Shas ont pour l'effet aussi d'augmentation de la conscience islamique en Palestine. Les insinuations de l'Ikhwan ont trouvé la réponse parmi les Palestiniens: Ikhwan défendait l'idée que le succès d'Israël était lié à leur dépendance à la religion alors que la raison d'échec des Palestiniens se trouvait dans l'éloignement de l'âme de la résistance et de l'Islam (Doyran 2008, 81).

Malgré sa croissance et son efficacité à travers ses activités et ses services sociaux; un certain mécontentement est resté au sein du mouvement d'Ikhwan. C'était à cause de certaines choses: L'Ikhwan avait refusé d'engager au combat contre l'occupation, son but était tout d'abord d'élever une génération Musulmane pour la résistance. Le combat était l'étape postérieure. Mais, les jeunes qui ont grandi dans les camps de réfugiés insistaient sur passage au djihad dès que possible. D’autre part, il y avait désormais parmi les membres du mouvement des jeunes élites qui étaient contre la direction traditionnelle. Cette nouvelle élite était éduquée mais par l'origine sociale, ils appartenaient à la classe inférieure. Cette nouvelle classe a dû sa mobilisation verticale à leur éducation. Glenn Robinson définit cette classe en montant, comme une «nouvelle classe moyenne ». En dernière analyse ces mécontentements ont devenu les catalyseurs en formation d'un cadre qui formerait Hamas. Le Djihad Islamique ${ }^{\mathrm{ii}}$ est né dans cette circonstance en 1980; les petits groups sont nés aussi à l'intérieur des Frères Musulmans, telles que: Al Takfir wa al- Hijra (L'Expiation et Le Vol Saint) et Tandhim al Djihad (L’Organisation de Djihad).

Le problème principal façonnait autour de deux idées: « Est-ce qu'il faut d'abord fonder une société ayant la conscience islamique ? » ou « Est-ce qu'il faut d'abord résister à l'occupation et la faire disparaître ? » En fin, le deuxième point de vue est devenu dominant et L'Intifada de Décembre 1987 a marqué un tournant dans l'histoire de la résistance Palestinienne et le Hamas est né. Intifada est un processus dans lequel le Hamas a nationalisé. À la suite du premier Intifada, le Hamas a transformé en un mouvement de la résistance islamique et nationale (Doyran 2008, 84). D’ailleurs, dans la charte de Hamas les trois sphères sont mentionnées qui ont lien avec la cause Palestinienne: Ceux sont la sphère Palestinienne, la sphère Islamique et la sphère Arabe (c'est-à-dire 'national') (Abu-Amr 1993, 12).

D’après Abu-Amr (1998), la montée du mouvement Hamas pendant l’Intifada a montré que cet évènement a bouleversé la balance des pouvoirs existants avant l'Intifada. L'Intifada avait fait visible le mouvement islamique et lui avait donné le pouvoir. Le mouvement islamique est parvenu à concurrencer avec les autres groups nationaux dans les territoires occupés.

Selon sa narration historique, Hamas a élaboré à travers quatre phases principales (Mishal \& Sela 2000, 20): 
1. L’année 1967-1976: La construction du noyau des Frères Musulmans à Gaza malgré le règlement oppressive Israélite.

2. L’année 1976-1981: L'expansion géographique à travers les associations professionnelles dans la Bande de Gaza et la Cisjordanie; la construction des institutions, notamment al Mujamma al Islami (Le Centre Islamique) et L’Université Islamique de Gaza.

3. L'année 1981-1987: L'influence politique à travers les fondations des mécanismes d'action et la préparation pour la lutte armée.

4. L'année 1987: La fondation du Hamas comme l'aile combattant des Frères Musulmans en Palestine et le lancement du djihad qui continue à nos jours.

\section{Un Moment Décisif: Al-Aqsa Intifada}

Le deuxième Intifada est une description juste des affrontements qui ont englouti les territoires occupés à partir de la fin du Septembre 2000. Cette fois, les épisodes étaient plus condensés, les meurtres plus brutaux, les réactions plus rapides et la couverture de media plus intense. (Hammami \& Tamari 2001, 5). À vrai dire, c'était une réaction d'un peuple qui ont souffrit aux sanctions implicites de status quo pendant des années. De ce point de vue, al Aqsa Intifada marque une transition d'un système de contrôle à un contrecoup répandue. Les contestations qui ont commencé avec la visite de Sharon sont étendus dans très court temps en tout Palestine. Le nombre des morts et des blessés ont augmenté d'un jour à l'autre. Partout, il y avait des manifestations et des convois.

Par opposition au premier Intifada, les Palestiniens qui vivent en Israël ont participé, ils aussi, à l'Intifada; ils ont fait des grèves générales dans les usines où ils travaillent. Israël a répondu aux manifestations violemment. Les soldats Israélites ont tiré des armes et ils ont tué plusieurs Palestiniens. Après la visite de Ariel Sharon à Haram al Sharif, les leaders de deux camps ont décidé de se réunir à Sharm Al Sheikh pour cesser la violence. À la fin des négociations, les camps ont décidé au cessez le feu; mais ce qui était important c'était en vrai, l'attitude du Hamas qui orientait les rues. La réaction du Hamas aux négociations de Sharm al Sheikh était négative:

\section{Voilà la réponse du Hamas aux décisions des négociations: On rejet tous les résultats des conversations! Al Aqsa Intifada n'est pas commencé pour les petites revendications. On appelle les Palestiniens à s'opposer aux négociations et a résistance » (Bayraktar 2007, 102).}

Avec cette déclaration, le Hamas a mis à terme sa stratégie de flexibilité. Il a rejeté les demandes d'Arafat sur le soutien aux négociations et il a commencé à mener un soulèvement armé et toutes sortes du combat. Donc, la réaction du Hamas n’était non seulement à Israël mais aussi à l'Autorité Palestinien. Cette colère à l'administration a augmenté entre les Palestiniens et en fin le gouvernement d'Arafat est transformé en un malade saignant.

Par rapport au premier Intifada, la religion a joué un rôle essentiel mobilisant et symbolique dans le courant Intifada. Cependant, la ferveur religieuse est devenue une dimension saillante d'Intifada qui a englouti la plupart du temps le conflit entier (Hamami \& Tamari 2001, 13). La bombe qui a explosé le 5 Mars 2001 à Hadera comme une réponse aux politiques d'Ariel Sharon avertissait que les kamikazes (dans le discours de Hamas: les opérations de martyr) sont retournés. De cette date, les kamikazes qui ont commencé en 1993 comme une stratégie de résistance ont marqué le deuxième Intifada. Jusqu'au 13 Septembre 2006, il y avait lieu 153 kamikazes pendant Al Aqsa Intifada. 80 l'incident des kamikazes sont mis au pratique par Hamas (Boran 2006, 105-6).

La stratégie de résistance qu’a mené Hamas contre celle de Fatah pendant le deuxième Intifada a assuré les déclinaisons importantes au mouvement du Hamas. La méthode est 
empruntée nationalement. Selon Hroub, chaque assassinat qui ciblait Hamas et chaque politique de pression contre Hamas ont grandi cette organisation dans les yeux des Palestiniens et cette situation a légitimé le mouvement. (Doyran 2008, 94). D'autre part, l'insistance de l'Autorité Palestinien sur la paix avec Israël et son silence pendant l'Intifada a rendu les Palestiniens de penser à une autre alternative. Edward Said (2004) avait bien réussi à devenir la voix des Palestiniens en définissant $\mathrm{Al}$ Aqsa Intifada «comme une réaction non seulement à Oslo, au processus d'Oslo, ni à Dennis Ross ou à Barak; mais aussi à un group des dirigeants Palestiniens qui n'ont rien avoir avec la responsabilité ». Tous ces circonstances ont préparé la victoire du Hamas aux élections de 2006.

Après le décès d'Arafat en 2004, Mahmoud Abbas est devenu le président. Dès qu'il est élu, il a fait une démarche pour la paix. Mais pour qu'il réussisse, la résistance armée doit être terminé. Le 19 Mars 2005, après les négociations entre tous les groupes Palestiniens, Palestine a déclaré le cessez le feu unilatéral et le peuple Palestinien a décidé d'aller aux élections qui n'étaient pas faits depuis 1996. Quand Hamas a expliqué qu'il participerait aux élections, les débats ont commencé. Par conséquent, les élections sont suspendues à l'année 2006 où Hamas a pris le pouvoir, à lui seul.

\section{Une Victoire Inattendu: Les élections de 2006}

Le processus de croissance du Hamas depuis Oslo s’est terminé avec une victoire qui a stupéfié le monde entier. Etant le seul opposant à Oslo, ayant un agenda social et appliquant les tactiques de lutte guérilla; Hamas s’est engagé dans la société Palestinienne en gagnant 72 des 132 sièges dans Le Conseil Législative Palestinien. Selon les rapports des observateurs internationaux; 77 $\%$ des Palestiniens ont voté et les élections ont lieu à la façon démocratique dans les territoires occupés.

Pour la première fois dans l'histoire contemporaine de Palestine, un groupe Islamique a pris la parole pour les Palestiniens. Comme était expliqué au début, aucun fait ne se réalise spontanément. Donc, il est possible d'affirmer ici que la victoire du Hamas est un fait préparé depuis longtemps. Il faut souligner que dans les temps du chômage et du manque des services sociaux à l'époque dégénéré d’OLP; c'était Hamas qui a offert aux Palestiniens des réseaux sociaux, les cliniques de santé etc. Depuis le premier Intifada, les Palestiniens étaient entrés dans une transformation sociale; soi religieuse soi politique. D'autre part, son opposition aux pratiques d'OLP qui ne pouvait pas trouver une solution à la question de Palestine a augmenté son pouvoir politique. La disparition du processus de paix avec Al Aqsa Intifada a aussi un effet dans l'augmentation du soutien au Hamas. La mort d'Arafat est l'un des facteurs en augmentation de la popularité du Hamas car Arafat jouait un rôle unificateur. La baisse du soutien au Fatah peut être expliquée avec la disparition de ce rôle (Altunışık 2006).

Hamas a pris le pouvoir le 26 Janvier 2006. Dès ce moment-là, la tension a commencé à augmenter; avec Fatah mais aussi avec Israël. Mahmoud Abbas avait expliqué qu'ils n'auront pas lieu dans le gouvernement que le Hamas fonderait. Donc, les deux groups sont entrés dans une collision du pouvoir. La tension qui s'accroitre entre le Hamas et le Fatah a terminé avec les dialogues qui sont faits à Mecque par la médiation de l'Arabie Saoudite le Février 2007. Le Hamas a accepté 7 ministériels au lieu de 9. Sur les dialogues, le Fatah a affirmé: "Ce marché ne fera que gagner le temps, sinon notre combat du pouvoir contre Hamas se prolongera. " Comme prévu, Israël a reçu le succès du Hamas avec le choc. Israël pensait que le Hamas gagnerait assez des sièges pour briser le tout pouvoir du Fatah, mais il n'avait jamais pensé qu'il serait le parti en majorité. La réponse d'Israël n'était pas en retard.

Le Premier Ministre intérim, Ehud Olmert a étonné dans la sixième conférence IsraëlEurope à Jérusalem le 6 Février: "Il n'y aura pas de reconnaissance d'un gouvernement Palestinien avec la participation ou sous le contrôle du Hamas » (Usher 2006). Actuellement, 
le discours de Hamas pendant les campagnes électorales n'était pas basé sur la destruction d'Israël; n'importe que soit son discours général; sa propagande était basée sur le combat avec l'illégitimité, la société ouverte, la fin au chômage et à la pauvreté (Aydın 2006). Le 11 Avril, le député Danni Yatom a déclaré que tous les ministères du gouvernement du Hamas étaient des cibles que Israël allait assassiner (Boran 2006, 186). Ensuite, Israël a mis sur l'agenda l'arrêt des aides à Palestine. Par conséquent, les pays dits démocratique, les Etats-Unis et l'Union Européen ont décidé de faire attendre les aides qui vont directement au gouvernement. D’ailleurs, Hamas qui est élu démocratiquement serait puni.

Le problème est que les pays occidentaux et Israël acceptent le Hamas comme une organisation terroriste. Mais, pour mieux comprendre la perspective, il faut noter ici que le Fatah aussi se trouvait dans la liste des organisations terroristes des Etats-Unis jusqu'aux négociations de Madrid en 1991. Être une organisation terroriste au Moyen Orient n'est pas conjugué avec le nombre des morts ou les activités qui sont réalisées; c’est conjugué surtout avec les autres dynamiques.

À cause d'embargo, la situation économique a empiré. L'Etat est venu au point de faillite dans court temps. C'étaient les hôpitaux qui sont influencés le plus. Les médicaments s'épuisaient d'un jour à l'autre. La Palestine était en train d'entrer dans une crise humanitaire. Israël pensait que l'administration se déferait au regard des Palestiniens. Bien plus, les explications du Ministre des Affaires Etrangères des Etats-Unis, Condoleezza Rice qui sont parus à Jérusalem Post le 11 Mai 2006 résumaient le but: «Au passé, les sources ont été donné à Abu Mazan (Mahmoud Abbas) car il s'est consacré à la paix. Le Hamas doit prendre la responsabilité pour son propre public » (Bayraktar 2007, 232). Mais au contraire pour les Palestiniens les vrais responsables de la situation dans laquelle ils se trouvaient sont les pays qui ont cessé leurs aides à Palestine.

Cette isolation et pression ont fait naitre une crise sérieuse. Le 17 Avril, un Palestinien s'est suicidé comme une réponse aux massacres d'Israël, avec une bombe à Tel Avive et il a tué 9 Israelites avec lui. Le gouvernement du Hamas a déclaré les condoléances et a ajouté que ces sont des contre-attaques inévitables contre les crimes que Israël a commis (Bayraktar 2007, 2278). Depuis ce jour jusqu'au Novembre 2006, les actions violentes ont ensanglanté les territoires Palestiniens. En 2006, Israël et Palestine se sont accordés sur un cessez le feu malgré que ce ne soit jamais un vrai cessez le feu. Un an après, l'aile militaire du Hamas, Izz al Din al Qassam Brigades a déclaré qu'il y n’aura plus les accords en accusant Israël de ne pas obéir aux accords.

Monte Palmer (2006) Professeur à l’Université Américain de Beyrouth, parle des six options pour Israël en traitant avec Hamas: autoriser le Hamas, détruire le Hamas, convergence, transformer les territoires occupés aux réservations Palestiniens, réoccupation et le transfert. Quant à Hamas, il compte 4 stratégies potentielles: résignation, modération, formation d'un gouvernement d'unité national et radicalisation.

Dans la dernière partie d'étude, on essaiera de mettre en place le discours des trois journaux turcs à propos des élections de la Palestine en 2006, avec la méthode d'analyse critique du discours.

\section{L'analyse des Données: La couverture du Hamas dans Trois Journaux Turcs}

Car le sens est construit par la société, donc la langue est un instrument qui reflète le sens. La langue comme un fait social et systématique est une partie de l'ordre symbolique. Dans ce contexte la langue et le système idéologique entrent en relation. Donc, il est possible de dire que dans les études de langue, les éléments sociales ont une place importante; même dans les études sociales; les éléments de langue ont la place importante. L'ordre symbolique qui se compose des idéologies s’identifie via la langue. Dans ce contexte, il sera possible d'arriver aux détails de l'ordre symbolique social en analysant la langue. 
Dans notre étude, les textes d'information sur le Hamas qui ont eu lieu dans la presse écrite entre les dates 23 Janvier 2006 et 30 Janvier 2006 seront analysés par l'analyse critique du discours. Par cette méthode, les values culturelles, les rapports du pouvoir (hégémonie), les idéologies, les identités et les faits sociaux variables qui ont préparé les conditions d'un ordre symbolique seront mis en évidence en partant des constructions linguales. Conséquemment, on essayera de montrer à la façon objective et systématique les structures implicites des textes traités en partant de leurs structures explicites linguales.

Car notre étude s'est fixée sur l'approche micro de Van Dijk; on a choisi la presse écrite pour l'analyse. On a choisi trois journaux turcs: Hürriyet, Birgün et Radikal. Les journaux sont choisis selon le positions idéologiques. Birgün est un journal qui s'identifie à gauche de l'éventail; Radikal se définisse comme un journal libéral. Quant à Hürriyet, c'est un journal le plus lu; soi-disant d’une tendance générale mais plus nationaliste.

\section{La quantité générale des données}

À la période de 23 Janvier 2006 et 30 Janvier 2006, trente-cinq informations sont parues dans quatre journaux. Birgün a donné lieu aux neuf informations du texte sur les élections en Palestine. Huit informations sont parues dans Radikal. Quant à Hürriyet, il était le journal qui avait séparé le moins de place à l'événement, cinq informations de texte. (Tableau 1)

Tableau 1. La Quantité Générale des Données

\begin{tabular}{|c|c|c|c|}
\hline Journal & Birgün & Hürriyet & Radikal \\
\hline \multicolumn{4}{|l|}{ Avant les } \\
\hline Élections & 2 & & \\
\hline \multicolumn{4}{|l|}{ Après les } \\
\hline Élections & 6 & & \\
\hline Total & 8 & & \\
\hline
\end{tabular}

\section{Les Manchettes}

\section{Janvier 2006}

« Hamas silah birakmayacak » Birgün (Hamas ne laissera pas les armes).

« Hamas'a karşı Barguti kartı » Radikal (Barguti contre Hamas).

\section{Janvier 2006}

« Filistinli kadınlar seçim yarışında » Birgün (Les femmes Palestiniens en compétition des élections).

\section{Janvier 2006}

« Artık sandıklar konuşacak » Birgün (C’est la tour des urnes de vote.

« En heyecanlı seçim » Radikal (Les élections le plus chaudes).

«Filistin'de bugün seçim var » «El Fetih imajdan kaybedecek » Hürriyet (Aujourd'hui les élections en Palestine- Fatah perdra le prestige).

\section{Janvier 2006}

« Bütün gözler Hamas’ta » Birgün (Tous les yeux sur Hamas).

« Ve Hamas sahneye çıtı... » Radikal (Hamas sur la scène).

«Filistin halkı kaderini oyladı » Hürriyet (Palestiniens votés pour destin). 


\section{Janvier 2006}

« Ortadoğu'da Hamas kilidi » Birgün, premier page en manchette, (Serrure du Hamas au Moyen Orient).

«Uluslararası toplumdan kaygı mesajları »Birgün, la manchette des pages internationaux, (Messages de crainte internationaux).

« Hamas büyük farkla galip » Radikal (Hamas gagnant avec un grand score).

« Kaleş iktidarda » Hürriyet, premier page, en manchette (Les armes au pouvoir).

« Hamas ihtilali » Hürriyet, la manchette des pages internationaux, (Coup du Hamas).

\section{Janvier 2006}

« Hamas'1 'ehlileştirme' çabaları » Birgün, (Les efforts pour apprivoiser Hamas).

« Hamas dünyayı böldü » Radikal (Hamas a divisé le monde).

« Erdoğan: Hamas'a firsat verilmeli » Radikal, la deuxieme manchette (Erdogan: Il faut donner l'occasion au Hamas).

«Kaleşi at baskısı » Hürriyet, la manchette des pages internationaux, (La pression de laisser les armes).

\section{Janvier 2006}

« El Fetih başkaldırıyor » Birgün, (Fatah se revolte).

«Gerilim artıyor: Filistin karmakarışık» Radikal, (La tension augmente: Palestine embrouillé).

« Ya kaleş ya para » Hürriyet, la manchette des pages internationaux, (Soi kalachnikov soi argent).

\section{Janvier 2006}

« Hamas'tan ABD’ye yeşil 1şık » Birgün, (Messages chauds aux Etats-Unis).

« Hamas'tan çağrı var » Radikal, (Un appel par Hamas).

Les manchettes sont les premiers indices montrant l'importance donnée par les journaux au sujet. La place de la manchette du sujet nous donne une idée pour comprendre l'attitude du journal. Après avoir examiné les manchettes sur les élections en Palestine, on voit qu'il n’y a pas des différences significatives. Cependant, il est possible de voir qu'il existe certaines différences entre les journaux. Leurs choix des topiques se diversifient. Par exemple, pour le 23 Janvier 2006, les manchettes des trois journaux sont différant bien que l'événement principal reste la même. On pourrait dire que Birgün a attribué beaucoup plus d'importance au rejet du Hamas sur ne pas laisser des armes alors que Radikal mentionne l'appel de Barguti. Les résultats des élections sont la manchette du premier page le 27 Janvier 2005. Parmi les trois journaux, c'est Hürriyet qui n'a pas attribué tant d'importance que les autres journaux. Il est aussi exceptionnel avec ses manchettes, il est le seul ayant une attitude le plus négative vers Hamas dans les manchettes.

\section{Les choix des mots et la rhétorique des informations}

On va mentionner dans cette partie, les mots qui sont le plus utilisés dans les textes d'information et qui attribuent un sens (négative/positive) au sujet de cette étude. Ce qu'on avait essayé de montrer depuis le début de cette étude que les textes d'informations changent notre façon de perception se montre en générale dans les choix des mots. Par exemple, le mot «l'organisation terroriste », pour donner la référence à l'acteur de l'information est répété plusieurs fois dans les textes. Car on n'a pas l'habitude de lire les textes mot par mot afin d'analyser les sens cachés derrière; cette référence prendra lieu dans nos têtes; une autre fois, 
quand on rencontre un article sur cet acteur, soudain on se rappellera lui comme on a appris par le journal: que le sujet de l'information est une organisation terroriste. C'est le cas pour notre sujet d'étude. Dans le tableau ci-dessous (Tableau 2), on a présenté huit mots qu'on trouve le plus expressives qui montrent que le choix des mots est un acte important.

Les façons d'utilisation des mots changent selon les journaux. Les mots «la mort, tuer, mourir » sont implémentés dans Radikal et Birgün dans la plupart des textes pour les Palestiniens qui sont tués par Israël. D’autre coté, dans Hürriyet le mot signifie les Israélites qui sont tués par le Hamas. Dans les autres journaux, le mot est utilisé avec les mots " organisation, mouvement » pour identifier Hamas; en lui attribuant des sens négatifs. Le nombre d'utilisation du mot « terreur » est la même dans trois journaux. Mais, il existe encore des différences d'attribution. Dans Birgün le mot se passe dans les paroles faites par les ministères Israélites, en d'autres termes; ce mot se trouve dans la partie des actions verbales de texte. Quant à Hürriyet, ce mot est utilisé encore pour créer un avis à propos du Hamas: "Umm Nidal qui est connu comme la mère des martyrs a filmé son fils alors qu'il irait aux opérations de terreur ». 28 Février 2005

«L'arme », c'est le mot le plus présenté dans les textes informatifs de chaque journal. Il signifie que le Hamas est un mouvement armé. Bien que les images ne soient pas incluses dans notre étude, il faut noter ici que dans les photos utilisées par les journaux il y a des gens, des enfants aussi, toujours avec les armes, particulièrement avec les kalachnikovs. C'est la raison pour laquelle la manchette de Hürriyet est « Les Kalachnikovs au pouvoir » en faisant référence aux membres du Hamas. "Est-ce que Hamas continuera à la terreur ? » demande Hürriyet, en pré-acceptant que Hamas soit une organisation terroriste.

« Radicale » est généralement utilisé avec les mots « organisation, mouvement, discours, ligne, perspective » pour identifier encore Hamas. D'après nous, l'implémentation du mot «Résistance » en majorité, donne une idée le plus fortement, à propos de la perspective du journal car ce qui est plus discuté sur Hamas est s'il est un mouvement de résistance ou un groupe de terroriste. Seulement c'est Hürriyet qui n’a pas présenté Hamas comme un mouvement de résistance. Au lieu de résistance, il a préféré d'utiliser «révolte» ou « terroristes » ou « kamikazes ». Birgün met l'accent souvent à la résistance armée du Hamas.

C’est Hürriyet, le seul qui a identifié Hamas avec Kamikazes. Dans la partie majoritaire des informations " Kamikazes " sont implémentés au lieu du mot Hamas: "L'organisation des Kamikazes », " La victoire des Kamikazes », " La mère des Kamikazes le Ministre du Hamas » etc. Quand on examine la structure du discours de Hürriyet, les résultats ne sont pas déclarés avec objectivité. Il n'a pas structuré son information avec un langage positif. Par contre, les phrases au sens négatifs dominent tous les textes. S'il y a un sens caché derrière les mots; selon Hürriyet le succès du Hamas n'apportera pas la paix au Moyen Orient, du même, le choix des Palestiniens est faux. Avec ses évaluations plus proches à Israël; le journal n’a pas pu assurer la balance entre deux camps.

Tableau 2. Les Mots Le Plus Utilisés

\begin{tabular}{lcccc}
\hline Journal & Birgün & Hürriyet & Radikal & Total \\
La mort/tuer & 4 & 4 & 3 & 11 \\
Mourir & & & & \\
Violence & 7 & 6 & 4 & 17 \\
Terreur & 12 & 12 & 4 & 28 \\
Arme & 28 & 26 & 22 & 76 \\
Radicale & 8 & 2 & 1 & 11 \\
Résistance & 14 & 3 & 10 & 27 \\
Résistance Armée & 2 & non & 4 & 6 \\
Kamikaze & non & 7 & non & 7
\end{tabular}


Par rapport à Hürriyet, les autres deux journaux Birgün et Radikal sont restés plus neutres. Le langage avec lequel ils ont construit leurs textes de l'information est sobre et plus pondéré.

\section{La couverture du bâton et de la carotte}

Dans notre revue des textes de l'information de la presse écrite, on a observé que le media Turc a adopté la politique du bâton et de la carotte. Ce politique reflet les conditions de transaction possible entre Israël et le Hamas si Hamas laisse les armes. Après les élections, Israël n’a pas voulu accepter le succès du Hamas, car selon lui, le Hamas est une organisation terroriste et qu'il ne peut pas accorder avec Hamas. Le media a poursuit la politique du «bâton » pour ne pas connaitre la légitimité du Hamas. Les textes adoptent une tonne menaçante qui excitent Hamas:

« Ni le monde ni Israël n'acceptera pas un gouvernement fondé par Hamas » 27 Janvier 2006, Hürriyet.

"La retrait d'Israël de Gaza l'été dernier avait ouvert une fenêtre à la paix. Les Palestiniens l'ont fermée en préfèrent Hamas dans les élections-ce que dit Tzipi Livni » 27 Janvier 2006, Hürriyet.

"Les Etats-Unis cessera les aides à Palestine si Hamas n’enferme pas son aile militaire-ce que dit George Bush » 29 Janvier 2006, Hürriyet.

«Un Hamasistan est fondé devant nos yeux-ce que dit Binyamin Netanyahu, c'est un tremblement de terre » 27 Janvier 2006, Radikal.

«Binyamin Netanyahu a demandé au monde cesser les aides à Palestine » 28 Janvier 2006, Radikal.

"Il est déclaré que Les Etats-Unis a donné une permission à Israël de ne pas connaitre Hamas en cas d'un succès aux élections » 24 Janvier 2006, Birgün.

"Olmert a déclaré qu'Israël s'accordera avec les Palestiniens et Mahmoud Abbas dans une seule condition d'enfermer les organisations terroristes » 27 Janvier 2006, Birgün

«Hitler aussi, il avait obtenu le pouvoir par les élections démocratiques-ce que dit Binyamin Netanyahu » 30 Janvier 2006, Birgün.

Conséquemment, le point principal de ce modèle est l'usage de media comme un instrument éducationnel qui vise redresser le Hamas vers au «droit chemin » (Rinnawi 2007). Pendant une semaine, les journaux ont parlé des conséquences et des réactions d'Israël en cas de l'élection du Hamas. Ils ont montré les «bâtons » pour, soi-disant « apprivoiser » Hamas en menaçant les Palestiniens avec la famine ou avec le manque des choses vitales. Ils sont devenus la voix des Etats-Unis et Israël, dans la plupart du temps. Ils ont donné lieus aux explications du Hamas, aux situations des Palestiniens; mais il y avait toujours déséquilibre.

\section{Les références attribuées à l'acteur des informations}

La description du Hamas est importante en premier lieu car elle peut montrer les évaluations implicites du Hamas. Tableau 3 liste les désignations par trois journaux pour Hamas. Généralement, les descripteurs du rôle comme «l'organisation », «le mouvement », «les 
militants » sont utilisés. Les autres désignations sont plus évaluatives: «les kamikazes », « controversable » etc. On a observé que Hürriyet a donné une mal référence au Hamas.

\section{La catégorisation schématique}

Dans cette section, on a examiné l'organisation des articles des textes (l'histoire, la résumé, les conséquences, les commentes etc.) sur le succès du Hamas. Notre observation a montré que la majorité des journaux locuteurs ne peut apprendre que l'événement principale et généralement, ils ne donnent pas l'information sur l'histoire, contexte ou les autres arrière-plans du problème. Le succès du Hamas (au sens négatif) est suffi car les values courant des informations se composent de la négativité, la violence et les spéculations. On a choisi le 27 Janvier où les conséquences exactes sont déclarées. (Tableau 4).

Tableau 3. Les descriptions stylistiques du Hamas

\section{Birgün (23-30 Janvier 2005)}

L'organisation radicale, le mouvement Islamique, Hamas se tirant avec Fatah au pouvoir, ...que Hamas est un group des terroristes, le mouvement radical Palestinien, Hamas qui participent aux élections pour la première fois, Hamas gagnant des élections, l'organisation islamique, un groupe de terreur qui vise annihiler Israël (Olmert), l'organisation radicale islamiste, Hamas sur le fond islamique et qui s'occupe majoritairement des affaires de charité, Le Mouvement Islamique de Resistance.

\section{Hürriyet (23-30 Janvier 2005)}

L'organisation radicale, l'organisation islamiste des kamikazes, le mouvement de résistance islamique, Hamas qui a arrangé des dizaines de suicides, les kalachnikovs, la partie des kalachnikovs, Hamas qui veut annihiler Israël et fonder un Etat Islamique en Palestine, « Hamasiens » qui se baladent avec des armes, l'organisation des kamikazes et des kalachnikovs Hamas dont le cible est d'effacer Israël et fonder un Etat Islamique, la partie de résistance, reforme et la diversité (Ismail Haniya), Hamas qui a interdit de danser à cause de danser est un péché, le gouvernement de terreur (Tzipi Livni), Hamas qui a renversé le gouvernement de Fatah, Hamas qui a tué des dizaines des Israelites avec des opérations de suicide, Hamas qui s'est consacré à l'annihilation d'Israël, radicale islamiste Hamas, le Mouvement de Resistance Islamique connu par Etats-Unis comme un groupe de terroristes, la partie politique qui prendra la parole à l'administration de l'Etat (R. T. Erdoğan), le gouvernement qui veut anéantir un pays qui est un ami et un allié de nous (Bush)

\section{Radikal (33-30 Janvier 2005)}

Le gagnant des élections Palestiniens, Hamas qui surveille de détruire Israël (Olmert), l'organisation de résistance armée, l'organisation terroriste qui appel pour briser Israël (Olmert), Hamas qui est en faveur de résistance armée contre Israël, Hamas qui veut adosser ses points de vus islamique et juridique (Mahmoud Abbas).

Tableau 4. Les catégories schématiques dans la Couverture de la Presse Turc le 27 Janvier 2005

\begin{tabular}{lccc}
\hline Journal & Birgün & Hürriyet & Radikal \\
\hline $\begin{array}{l}\text { Manchette } \\
\text { Premier }\end{array}$ & $\checkmark$ & $\checkmark$ & $\checkmark$ \\
Paragraphe & $\checkmark$ & $\checkmark$ & $\checkmark$
\end{tabular}


Evénement

Principale

Background

Histoire

Contexte

Conséquences

Réactions

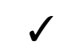

non

non

non

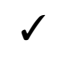

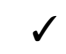

$\begin{array}{cc}\text { non } & \text { non } \\ \text { non } & \text { non } \\ \text { non } & \text { non } \\ \checkmark & \text { non } \\ \checkmark & \checkmark\end{array}$

\section{Résultats}

Notre analyse du discours fournit des évidences que les textes d'information sur Hamas dans la presse Turque a employé une couverture de « délégitimation » avec une exception. Au regard du langage des textes, on a trouvé que les journaux ont pris des manières menaçantes et négatives en structurant leurs textes d'information. Dans la plupart des textes les mots qui réfèrent à la violence et la mort sont dominants. La manière dont les journaux traitent des résultats des élections fait croire au public que la paix est impossible tant que le Hamas est au pouvoir au Gaza. Ceci est corroboré par la couverture des déclarations des dirigeants Israéliens qui désignent le Hamas comme une organisation terroriste et en expliquant pourquoi les résultats sont inacceptables.

$\mathrm{Au}$ regard du contenu des textes d'information les définitions du résultat des élections donnent l'idée que le choix des Palestiniens empirera la situation en Palestine, telles que: « Le coup du Hamas, Hürriyet », "Le sens commun: Inquiétude, Birgun », "C’est un tremblement de terre, Radikal ». C’est le même cas quant à la référence à l'acteur des informations: Négative en majorité (voir la Tableau 3). Les journaux n'ont pas eu besoin de donner des informations sur le contexte historique ou actuel, ni sur Hamas ni sur le conflit Israélo-palestinien.

Par conséquent, on est arrivé à la conclusion que ces trois journaux n’ont pas reflété les nouvelles sur Hamas à la façon pondérée. Notre étude a montré que les perspectives idéologiques des journaux, la façon d'interpréter le monde, leurs structurations ont joué un rôle important qu'aux étapes des sélections des informations, de la construction des textes d'information et de la présentation.

\section{Conclusion}

Le Hamas étant élu par les façons démocratiques est l'un des acteurs importants qui orient la politique de Palestine. Malgré la dialectique entre son passé et sa situation d'aujourd'hui, le mouvement est devenu un déterminant sur le futur de Palestine. Les conditions qui ont ouvert la voie pour le Hamas sont en relation avec l'occupation d'Israël et avec la résistance du public. Le Hamas comme un mouvement de résistance est une conséquence de cette occupation Israélite. Les conditions sociopolitiques dans lesquelles les Palestiniens se trouvent ont préparé non seulement la révélation de ce mouvement mais aussi elles ont déterminé le discours, la méthode de résistance et les tactiques qu'utilise le Hamas. Le processus d'Oslo et le deuxième Intifada ont augmenté le prestige du Hamas alors qu'OLP perdait ses partisans. Sa structure d'organisation, ses traditions qui viennent des Frères Musulmans ont assuré de gagner l'acceptation des Palestiniens en court temps. Son engagement islamique est un autre facteur fort en cette acceptation car tout le Moyen Orient passait un processus d'Islamisation à cette époque-là.

Hamas a gagné « une victoire » aux élections de 2006 contre OLP qui est connu jusqu’à là la seule force politique de Palestine. Au lieu de respecter au choix des Palestiniens; les EtatsUnis, l’Union Européen et Israël et quelques régimes Arabes ont compté le résultat des élections 
comme un prétexte pour appliquer l'embargo et la pression aux Palestiniens. L'opinion publique internationale est aussi bien orienté; avec l'arme le plus subtile: Le media. Dès que les résultats sont expliqués, les leaders du monde ont commencé à donner des paroles aux journaux pour qu'ils les transmettent au public dès que possible. Le media a travaillé fort comme s'il essayait de réaliser un consensus pour délégitimer les résultats des élections. Les paroles d'Olmert, de Bush, de Netanyahu étaient publiées dans chaque journal alors que celles de Haniya, de Zahhar sont données superficiellement. Comme Edward Said avait observé pendant le deuxième Intifada qu'il n'y avait qu'un article pro-Palestinien, mais des dizaines d'eux pro-Israélite; on a aussi essayé de faire une observation sur les élections de 2006 afin de trouver s'il a raison.

Les résultats ont montré que les perceptions des locuteurs sont manipulées à travers l'usage duquel appel Gans (1979) « les informations d'ordre et désordre ». Tandis que les informations d'ordre réfèrent aux perspectives positives dans une façon de présenter favorablement le groupe qui se trouve derrière les informations comme un acteur; contrairement l'information de désordre insiste sur les perspectives négatives et présentent les groupes dans les informations à la manière défavorable en attribuant leurs attitudes comme destructive et menaçant.

Après avoir examiné les journaux qu'on a choisis, les évidences ont montré que la présentation du Hamas à la suite des élections est négative. Hamas est représenté comme une menace de sécurité à Israël et au monde; les Etats-Unis et l’Union Européen ont participé à ces explications. Par l'aide des journaux, ces explications ont trouvé leurs places dans les têtes du public qui nous amène à l'argumentation que le media joue un rôle important dans le processus de formation de l'opinion publique. On a trouvé que la couverture des nouvelles dans trois journaux Turcs sur Hamas se compose des informations de désordre et qu'elle est anti-Hamas en générale; faisant une couverture de de-légitimation.

D’autre part, on a aussi trouvé des usages de la description stéréotypique dans les journaux. Par exemple, dans la plupart des textes de l'information, le coté islamiste et les opérations des suicides sont beaucoup cités. Cette étude a aussi montré qu'il existe un déséquilibre en présentation de deux acteurs des informations. Les sources utilisées sont de perspective Israélite; donc il y a aussi en problème de neutralité.

Le processus vécu après les élections de Palestine a montré ce qui est souhaité n’es pas une démocratie, mais un désir de voir les gouvernements qui peuvent servir à certains' intérêts. Donc, Hamas n'a pas de chance d'être accepté ni par Israël ni par Etats-Unis car il n’a servi pas les intérêts d'eux. 


\section{BIBLIOGRAPHIE}

Abu-Amr Z. (1993). "Hamas: A Historical and Political Background”. Journal of Paletsine Studies 22/4 (1193) 5-19.

Abu-Amr Z. (1998). Batı Şeria ve Gazze'de İslami Direniş. İstanbul 1998.

Altunışık M. (2006). Le discours fait dans le panel "Palestine après les élections". La Fondation SETA, https://www.setav.org/secimler-sonrasi-filistin/ consulté sur 28.09.2018

Aydın M. (2006). Le discours fait dans le panel "Palestine après les élections". La Fondation SETA, https://www.setav.org/secimler-sonrasi-filistin/ consulté sur 28.09.2018

Bayraktar B. (2007). Hamas. İstanbul 2007.

Bekalu M. A. (2006). "Presupposition in News Discourse”. Discourse and Society 17/3 (2006) 147-172.

Boran Y. (2006). Geçmişten Günümüze El Fetih ve Hamas. İstanbul 2006.

Cheek J. (2004). “At The Margins? Discourse Analysis and Qualitative Research”. Qualitative Health Research 14/8 (2004) 1140-1150.

Detrie C., Siblot P., Vrine B. (2001). Termes et Concepts pour l'Analyse du Discours. Paris 2001.

Doyran E. (2000). Bir Direniş Hareketi Olarak Hamas. Ankara 2000.

Dursun Ç. (2004). "Haberde Gerçekliğin İnşa Edilmesi Ne Demektir?. Ed. Ç. Dursun. Haber, Hakikat, İktidar İlişkisi (2009). Ankara.

Gans H. J. (1979). Deciding What's News: A Study of CBS Evening News, NBC Nightly News, Newsweek, and Time. Illinois 1979.

Ginneken J. V. (1998). Understanding Global News- A Critical Introduction. London 1998.

Hammami R. \& Tamari, S. (2001). “The Second Uprising: End or Beginning?”. Journal of Palestine Studies 30/2 (2001) 5-25.

Karakır İ. A. (2006). "Rising Political Islam: Is It a Matter of Ideology or Pragmatism?”. Turkish Policy Quarterly 5/4 (2006) 85-94.

Legrain F. (1997). “HAMAS: Legitimate Heir of Palestinian Nationalism?”. Ed. J. L. Esposito. Political Islam, Revolution, Radicalism or Reform? (1997). London.

Mishal S. \& Sela A. (2000). The Palestinian Hamas: Vision, Violence and Coexistance. New York 2000.

Palmer M. (2006). "Israel and Hamas: Problems and Options". Document non publié au congrès de Fondation pour Etudes du Moyen Orient et des Balkans.

Purvis T. \& Hunt A. (1993). "Discourse, Ideology, Discourse, Ideology, Discourse...”. The British Journal of Sociology 44/3 (1993) 473-499.

Rinnawi K. (2007). "De-legitimization of Media Mechanismes: Israeli Press Coverage of the Al Aqsa Intifada”. International Communication Gazete 69/2 (2007) 149-178.

Said E. W. (2004). Yeni Binyllda Filistin Sorunu. İstanbul 2004.

Usher G. (2006). “Hamas Risen”. Middle East Report 238 (2006).

Ülkü G. (2004). “Söylem Çözümlemelerinde Yöntem Sorunu ve Van Dijk Yöntemi”. Ed. Çiler Dursun. Haber, Hakikat, İkitdar İlişkisi. (2004). Ankara.

Van Dijk T. (1988a). News As Discourse. London 1988.

Van Dijk T. (2005). "Söylemin Yapıları ve İktidarın Yapıları". Ed. M. Küçük, Medya, İktidar, İdeoloji. (2005). Ankara.

Van Dijk T. (2006). “Discourse and Manipulation”. Discourse and Society 17/3 (2006) 359-383.

Van Dijk T. (1988b). News Analysis. London 1988.

Voll J. (2006). "Hamas: Past and Present”. The Palestine Center Reports (2006). 
